\title{
Editorial: Bacterial Cell Wall Structure and Dynamics
}

\author{
Tobias Dörr ${ }^{1,2 \dagger}$, Patrick J. Moynihan ${ }^{3 \dagger}$ and Christoph Mayer ${ }^{4 *}$ \\ ${ }^{1}$ Department of Microbiology, Weill Institute for Cell and Molecular Biology, Ithaca, NY, United States, ${ }^{2}$ Cornell Institute of \\ Host-Microbe Interactions and Disease, Cornell University, Ithaca, NY, United States, ${ }^{3}$ School of Biosciences, Institute of \\ Microbiology and Infection, University of Birmingham, Birmingham, United Kingdom, ${ }^{4}$ Department of Biology, \\ Interfaculty Institute of Microbiology and Infection Medicine Tübingen, University of Tübingen, Tübingen, Germany
}

Keywords: peptidoglycan, cell wall, autolysin, PG recycling, turnover

OPEN ACCESS

Edited by:

Kürşad Turgay,

Max-Planck-Gesellschaft, Germany

Reviewed by:

Peter Graumann,

University of Marburg, Germany

Leendert Hamoen,

University of Amsterdam, Netherlands

${ }^{*}$ Correspondence:

Christoph Mayer

christoph.mayer@uni-tuebingen.de

tORCID:

Tobias Dörr

0000-0003-3283-9161

Partick J. Moynihan

0000-0003-4182-6223

Christoph Mayer

0000-0003-4731-4851

Specialty section:

This article was submitted to

Microbial Physiology and Metabolism,

a section of the journa

Frontiers in Microbiology

Received: 25 July 2019

Accepted: 20 August 2019

Published: 04 September 2019

Citation:

Dörr T, Moynihan PJ and Mayer C (2019) Editorial: Bacterial Cell Wall

Structure and Dynamics.

Front. Microbiol. 10:2051.

doi: 10.3389/fmicb.2019.02051

\section{Editorial on the Research Topic}

\section{Bacterial Cell Wall Structure and Dynamics}

The bacterial cell wall is a complex, mesh-like structure that in most bacteria is essential for maintenance of cell shape and structural integrity. Historically, the cell wall has been of intense research interest due to its necessity for most bacteria and absence from the eukaryotic realm, positioning it as an ideal target for some of our most powerful antibiotics (Schneider and Sahl, 2010). In addition, bacterial cell wall fragments can have immunostimulatory and cytotoxic properties and thus play important roles in pathogenesis and disease (Goldman et al., 1982; Fleming et al., 1986; Royet et al., 2011; Sorbara and Philpott, 2011; Jutras et al., 2019).

The cell wall consists mainly of peptidoglycan (PG), a mesh of polysaccharide strands (composed of a poly-[ $\mathrm{N}$-acetylglucosamine (GlcNAc)- $\mathrm{N}$-acetylmuramic acid (MurNAc)] backbone) crosslinked via short peptide bridges attached to the MurNAc residues (Vollmer et al., 2008a). PG is synthesized on the external face of the cytoplasm. Synthesis steps include cytoplasmic generation of the lipid-linked disaccharide-pentapeptide precursor lipid II, translocation of lipid II to the outside of the cell by flippases (MurJ and/or Amj); and finally assembly of the cell wall by penicillin-binding proteins (PBPs) and Shape, Elongation, Division, and Sporulation (SEDS) proteins (Ruiz, 2008; Typas et al., 2011; Meeske et al., 2015, 2016; Cho et al., 2016; Taguchi et al., 2019). The assembly process can be further subdivided into polymerization of the GlcNAc-MurNAc-pentapeptide via glycosyltransferase reactions catalyzed by class A PBPs and SEDS proteins, and crosslinking of the peptide sidestems into a tight meshwork by class A and B PBPs and L,D-transpeptidases in a not (yet) fully-understood manner (Zhao et al., 2017). In addition, the PG mesh can be decorated with secondary cell wall polymers, such as wall teichoic acids (polyol-phosphate polymers) or capsule polysaccharides that are covalently attached to PG (Rajagopal and Walker, 2017). In the case of mycobacteria, layers of polysaccharides and long-chain lipids are added to the PG layer, making the cell wall structure even more complex (Jankute et al., 2015).

While the cell wall must be rigid enough to maintain high intracellular pressures and withstand environmental assaults, it also needs to be flexible enough to allow for cellular expansion. In addition to synthesis functions, the cell wall is thus also constantly broken down, turned over, and remodeled (Park and Uehara, 2008; Reith and Mayer, 2011; Mayer et al., 2019). This is accomplished by a poorly-understood, remarkable group of enzymes that collectively can cleave and/or modify a variety of PG structures. So-called "autolysins," for example, are a functionally 
diverse group of enzymes that cut PG crosslinks (endopeptidases), peptide sidestems (amidases, carboxypeptidases), or the sugar backbone (muramidases, lytic transglycosylases) (Scheurwater et al., 2008; Vollmer et al., 2008b). PG-acetyltransferases "decorate" MurNAc backbone structures with acetyl residues, imparting increased lysozyme resistance (Moynihan and Clarke, 2011). L,D-transpeptidases (Mainardi et al., 2008) orchestrate D-amino acid (DAA) exchange reactions that can replace terminal $\mathrm{D}$-Ala residues with a variety of alternative DAAs (Cava et al., 2011); this can be exploited to label PG with fluorescent compounds (Kuru et al., 2012). Many of these systems fulfill important functions such as daughter cell separation, sacculus expansion during growth, insertion of macromolecular trans-envelope protein complexes, and PG recycling (Scheurwater and Burrows, 2011; Vollmer, 2012; Johnson et al., 2013).

Due to its importance for bacterial survival and the many open questions concerning mechanistic details of synthesis and turnover, the cell wall remains at the center of a large number of active research programs. The last decade in particular has seen a resurgence in interest in the bacterial cell walla Pubmed search with the keywords "peptidoglycan synthesis bacteria" reveals a total of 7,762 publications, of which 3,532 (45\%) were published in the last 10 years. This renewed interest has been fueled by novel imaging techniques (super-resolution imaging and the development of live cell wall stains) and by new revelations of processes that had been thought to be wellunderstood for decades. Some recent examples include the finding that the class A "penicillin-binding proteins" (aPBPs) require outer membrane cofactors for in vivo function (ParadisBleau et al., 2010; Typas et al., 2010), and that RodA/FtsW possess glycosyltransferase activity (Taguchi et al., 2019). At the same time, the cell wall remains a highly attractive target for antibiotic development, which has in the last decade become ever more important due to the rise in antibiotic resistance development.

In this special topic issue, we explore some new developments in the realm of bacterial cell wall biology. This collection of articles touches upon several cornerstones of PG research, with contributions focusing on the cell wall as a target for novel antibiotics, and aspects of its synthesis, turnover and modification.

\section{THE CELL WALL AS A TARGET FOR ANTIBIOTIC DISCOVERY}

In a bioinformatics tour-de-force, Jukič et al. describe novel inhibitors of UppS, an isoprenyl transferase enzyme that catalyzes a critical step in the biosynthesis of the lipid carrier molecule undecaprenol pyrophosphate (UPP). UPP is essential for the translocation of the PG precursor lipid II and other extracellular polysaccharides and thus constitutes a promising target for a novel class of cell envelope antibiotics. These inhibitors were identified by virtual docking models that predicted molecule binding based on UppS crystal structures and their interaction with a known inhibitor, bisphosphonate BPH-629. This clever approach resulted in the identification of several inhibitors, one of them with $\mu \mathrm{M}$ range inhibitory activity.

Drug-resistant Mycobacterium tuberculosis strains are a major global threat that is not being adequately met with current drug discovery efforts. In their review article, Catalão et al. describe the history of peptidoglycan-targeting drugs and their use in mycobacteria. The authors provide a potential path forward by discussing recent advances such as therapies using $\beta$ lactam/ $\beta$-lactamase inhibitor combinations and the use of phage endolysins for the treatment of mycobacterial infections.

\section{CELL WALL SYNTHESIS AND ARCHITECTURE}

Using X-ray crystallography and liquid state NMR, Maya-Martinez et al. investigate the structure-function relationships of PBP4 of Staphylococcus aureus, a class C PBP that unexpectedly has no PG hydrolase (D,D-peptidase) activity, but only transpeptidase activity. S. aureus is characterized by a very high degree of PG cross-linking and PBP4 apparently plays a major role in this hyper-crosslinking. The authors show transpeptidase activity of PBP4 with disaccharide peptides in vitro, producing dimeric, multimeric, and cyclic products. Structural studies with an active site mutant (S75C) revealed potential binding sites for the donor and acceptor stem peptides involved in the transpeptidation reaction.

Hottmann et al. report on peptidoglycan metabolism in the oral Gram-negative pathogen Tannerella forsythia (Phylum Bacteroidetes). T. forsythia depends on an exogeneous supply of the cell wall sugar $N$-acetylmuramic acid (MurNAc), as it lacks genes generally essential for bacteria for de novo synthesis of the peptidoglycan precursor UDP-MurNAc. A pathway for the catabolism of MurNAc involving a MurNAc-6 kinase (MurK) and a MurNAc-6P hydrolase (MurQ etherase) was established in T. forsythia, which counteracts a proposed cell wall synthesis pathway that utilizes salvaged MurNAc from the medium. Accordingly, a mutant in murK exhibited increased tolerance to low external MurNAc concentrations, presumably since blocking MurNAc degradation enhances peptidoglycan precursor synthesis.

The exact in vivo architecture of PG is poorly understood. Li et al. used Atomic Force Microscopy (AFM) for a detailed study of PG architecture, particularly at the septum, in $B$. subtilis. Surprisingly, B. subtilis undergoes significant changes in thickness and overall cell wall architecture in different growth phases. Li et al. were also able to isolate and image septa at varying stages of completion, visualizing the PG dynamics of septal closure at high resolution.

In a thought-provoking perspective article, Vincent et al. present a hypothesis for the evolutionary origins of the unique mycobacterial cell wall through a series of horizontal gene transfers. They support their argument by observing the distribution of key cell-wall biosynthetic enzymes across the order, which suggests that the arabinogalactan components pre-date the outer membrane and virulence related lipids. In their article, the authors propose an experiment whereby the 
evolutionary origins of the leaflet could be tested by attempting to reconstruct the mycobacterial cell wall in an Actinobacterium that currently lacks this feature.

\section{CELL WALL TURNOVER AND MODIFICATION}

Duchêne et al. describe new phenotypes for endopeptidase mutants in Lactobacillus plantarum. The mechanisms of regulation and physiological functions of cell wall lytic enzymes are still poorly understood, particularly in non-model organisms. L. plantarum is an ideal system to study PG hydrolase phenotypes due to its relatively small number of PG lytic enzymes (a "mere" twelve!). The authors carefully dissect the cell biological consequences of the loss of L. plantarum's endopeptidases and assign new putative functions to these enzymes. This study thus lifts the curtain on endopeptidase function in a Grampositive non-model organism, which is of particular importance given the high level of redundancy of PG lytic enzymes in many model bacteria, which ordinarily makes gene-phenotype association difficult.

During cell wall turnover in the Gram-positive pathogen $S$. aureus, the MurNAc-GlcNAc disaccharide is released from PG by the major autolysin Atl and its components eventually reused for PG biogenesis. Kluj et al. report on the fate of this disaccharide, which is taken up and is concomitantly phosphorylated by a phosphotransferase system (PTS) transporter. In order to facilitate PG recycling, the product MurNAc-6P-GlcNAc is split intracellularly by a novel phospho-glycosidase (MupG), constituting the first characterized representative of a novel class of phospho-muramidase enzymes distributed mainly within the Firmicutes bacteria.

Hager et al. report on an intriguing mode of attachment used by some bacteria (e.g., Bacillus anthracis and Paenibacillus alvei) to bind cell-surface proteins to the cell envelope: pyruvylated secondary cell wall polymers act as high-affinity ligands for binding. In this study, the enzymatic pathway leading to the synthesis of pyruvylated disaccharide repeats, [-4-betaGlcNAc-1,3-(4,6-Pyr)-beta-ManNAc-1-], of the $P$. alvei cell wall polymer was reconstituted. The reconstitution involved recombinant $\mathrm{CsaB}$ enzyme, catalyzing the attachment of a pyruvate to position 4 and 6 of ManNAc in the lipid-linked precursor molecule.

Devine provides a concise mini-review about the phosphate starvation regulation in the Gram-negative E. coli and the Grampositive $B$. subtilis. In both organisms, phosphate limitation is sensed by the two-component system PhoPR. However, the mechanisms controlling the Pho response differ. In Bacillus subtilis, phosphate-limitation response is linked with wall teichoic acid metabolism. PhoR activity is controlled by biosynthetic intermediates of WTA metabolism, which either promotes or inhibits autokinase activity. In E. coli, phosphate is sensed directly through substrate-responsive conformational changes in a phosphate transporter.

Vermassen et al. give a comprehensive overview of the biochemistry and in vivo cleavage activity of PG lytic enzymes.
This review highlights the "mix and match" approach that many cell wall lytic enzymes have undergone, combining different PG cleavage catalytic functions (e.g., lytic transglycosylase and peptidase activity) within the same enzyme.

The unique chemical nature of PG allows it to act as a potent signaling molecule. Irazoki et al. provide an overview of the process of PG release across a broad range of bacteria and PG sensing by a wide range of hosts. The authors highlight the multiplicity of systems to generate and sense bacterial PG and suggest that there is still a great deal to be learned about the sensing of these important molecules. They conclude that this field will be driven by the development and application of new analytical technologies to identify novel PG receptors.

Peptidoglycan recycling among many Gram-negative bacteria is achieved through a core pathway of degradation, recovery and recycling. In some pathogenic Neisseria, the recycling system is partially defective, which leads to an increase in the release of immunostimulatory PG fragments. In their review article, Schaub and Dillard discuss some of the differences between Neisserial PG turnover and other, more intensively studied bacteria such as E. coli. They conclude by proposing Neisseria sp. as an attractive model system for the study of cell wall growth and turnover due to their lower number of cell wall-active enzymes, variation in cell shape, and natural competence.

In addition to variations in glycan composition and stempeptide composition, $\mathrm{PG}$ can also be $\mathrm{O}$-acetylated at the C6 of MurNAc, or, less frequently, GlcNAc. Sychanta et al. provide an overview of recent advances in understanding the biochemistry of O-acetyltransferase systems in Gram-positive and Gram-negative bacteria. They also discuss current efforts at understanding the impact of inhibiting these systems and address unanswered biological questions such as the source of acetate for wall modification.

Bacterial cell wall biology remains a major frontier, both in our quest to develop a profound understanding of fundamental microbiology and to discover novel compounds that may be used to treat infections caused by antibiotic resistant bacteria. We hope that this special issue further advances this frontier and inspires additional exploration-peptidoglycan is, in many ways, still as mysterious as it was 7,762 publications ago.

\section{AUTHOR CONTRIBUTIONS}

All authors listed have made a substantial, direct and intellectual contribution to the work, and approved it for publication.

\section{FUNDING}

Research in the TD lab was supported by National Institutes of Health (NIH) grants R01AI143704 and R01GM130971. PM was supported by a BBSRC David Phillips Fellowship (grant BB/S010122/1). CM acknowledges financial support by the German research foundation (DFG: grants MA2436/7, SFB766-A15, GRK1708-B2, and TRR261-A06) and the government of the state of Baden-Württemberg (MWK-Glycobiology/Glycobiotechnology). 


\section{REFERENCES}

Cava, F., de Pedro, M. A., Lam, H., Davis, B. M., and Waldor, M. K. (2011). Distinct pathways for modification of the bacterial cell wall by non-canonical D-amino acids. EMBO J. 30, 3442-3453. doi: 10.1038/emboj.2011.246

Cho, H., Wivagg, C. N., Kapoor, M., Barry, Z., P., Rohs, D. A., Suh, J. A., et al. (2016). Bacterial cell wall biogenesis is mediated by SEDS and PBP polymerase families functioning semi-autonomously. Nat. Microbiol. 1:16172. doi: 10.1038/nmicrobiol.2016.172

Fleming, T. J., Wallsmith, D. E., and Rosenthal, R. S. (1986). Arthropathic properties of gonococcal peptidoglycan fragments: implications for the pathogenesis of disseminated gonococcal disease. Infect. Immun. 52, 600-608.

Goldman, W. E., Klapper, D. G., and Baseman, J. B. (1982). Detection, isolation, and analysis of a released Bordetella pertussis product toxic to cultured tracheal cells. Infect. Immun. 36, 782-794.

Jankute, M., Cox, J. A., Harrison, J., and Besra, G. S. (2015). Assembly of the mycobacterial cell wall. Annu. Rev. Microbiol. 69, 405-423. doi: 10.1146/annurev-micro-091014-104121

Johnson, J. W., Fisher, J. F., and Mobashery, S. (2013). Bacterial cell-wall recycling. Ann. N. Y. Acad. Sci. 1277, 54-75. doi: 10.1111/j.1749-6632.2012.06813.x

Jutras, B. L., Lochhead, R. B., Kloos, Z. A., Biboy, J., Strle, K., Booth, C. J., et al. (2019). Borrelia burgdorferi peptidoglycan is a persistent antigen in patients with Lyme arthritis. Proc. Natl. Acad. Sci. U.S.A. 116, 13498-13507. doi: 10.1073/pnas.1904170116

Kuru, E., Hughes, H. V., Brown, P. J., Hall, E., Tekkam, S., Cava, F., et al. (2012). In situ probing of newly synthesized peptidoglycan in live bacteria with fluorescent D-amino acids. Angew. Chem. Int. Ed Engl. 51, 12519-12523. doi: 10.1002/anie.201206749

Mainardi, J. L., Villet, R., Bugg, T. D., Mayer, C., and Arthur, M. (2008). Evolution of peptidoglycan biosynthesis under the selective pressure of antibiotics in Gram-positive bacteria. FEMS Microbiol. Rev. 32, 386-408. doi: 10.1111/j.1574-6976.2007.00097.x

Mayer, C., Kluj, R. M., Muhleck, M., Walter, A., Unsleber, S., Hottmann, I., et al. (2019). Bacteria's different ways to recycle their own cell wall. Int. J. Med. Microbiol. doi: 10.1016/j.ijmm.2019.06.006. [Epub ahead of print].

Meeske, A. J., Riley, E. P., Robins, W. P., Uehara, T., Mekalanos, J. J., Kahne, D., et al. (2016). SEDS proteins are a widespread family of bacterial cell wall polymerases. Nature 537, 634-638. doi: 10.1038/nature19331

Meeske, A. J., Sham, L. T., Kimsey, H., Koo, B. M., Gross, C. A., Bernhardt, T. G., et al. (2015). MurJ and a novel lipid II flippase are required for cell wall biogenesis in Bacillus subtilis. Proc. Natl. Acad. Sci. U.S.A. 112, 6437-6442. doi: 10.1073/pnas.1504967112

Moynihan, P. J., and Clarke, A. J. (2011). O-Acetylated peptidoglycan: controlling the activity of bacterial autolysins and lytic enzymes of innate immune systems. Int. J. Biochem. Cell Biol. 43, 1655-1659. doi: 10.1016/j.biocel.2011.08.007

Paradis-Bleau, C., Markovski, M., Uehara, T., Lupoli, T. J., Walker, S., Kahne, D. E., et al. (2010). Lipoprotein cofactors located in the outer membrane activate bacterial cell wall polymerases. Cell 143, 1110-1120. doi: 10.1016/j.cell.2010.11.037

Park, J. T., and Uehara, T. (2008). How bacteria consume their own exoskeletons (turnover and recycling of cell wall peptidoglycan). Microbiol. Mol. Biol. Rev. 72, 211-227. doi: 10.1128/MMBR.00027-07

Rajagopal, M., and Walker, S. (2017). Envelope structures of gram-positive bacteria. Curr. Top. Microbiol. Immunol. 404, 1-44. doi: 10.1007/82_2015_5021
Reith, J., and Mayer, C. (2011). Peptidoglycan turnover and recycling in Gram-positive bacteria. Appl. Microbiol. Biotechnol. 92, 1-11. doi: 10.1007/s00253-011-3486-x

Royet, J., Gupta, D., and Dziarski, R. (2011). Peptidoglycan recognition proteins: modulators of the microbiome and inflammation. Nat. Rev. Immunol. 11, 837-851. doi: $10.1038 /$ nri3089

Ruiz, N. (2008). Bioinformatics identification of MurJ (MviN) as the peptidoglycan lipid II flippase in Escherichia coli. Proc. Natl. Acad. Sci. U.S.A. 105, 15553-15557. doi: 10.1073/pnas.0808352105

Scheurwater, E., Reid, C. W., and Clarke, A. J. (2008). Lytic transglycosylases: bacterial space-making autolysins. Int. J. Biochem. Cell Biol. 40, 586-591. doi: $10.1016 /$ j.biocel.2007.03.018

Scheurwater, E. M., and Burrows, L. L. (2011). Maintaining network security: how macromolecular structures cross the peptidoglycan layer. FEMS Microbiol. Lett. 318, 1-9. doi: 10.1111/j.1574-6968.2011.02228.x

Schneider, T., and Sahl, H. G. (2010). An oldie but a goodie-cell wall biosynthesis as antibiotic target pathway. Int. J. Med. Microbiol. 300, 161-169. doi: 10.1016/j.ijmm.2009.10.005

Sorbara, M. T., and Philpott, D. J. (2011). Peptidoglycan: a critical activator of the mammalian immune system during infection and homeostasis. Immunol. Rev. 243, 40-60. doi: 10.1111/j.1600-065X.2011.01047.x

Taguchi, A., Welsh, M. A., Marmont, L. S., Lee, W., Sjodt, M., Kruse, A. C., et al. (2019). FtsW is a peptidoglycan polymerase that is functional only in complex with its cognate penicillin-binding protein. Nat. Microbiol. 4, 587-594. doi: 10.1038/s41564-018-0345-x

Typas, A., Banzhaf, M., Gross, C. A., and Vollmer, W. (2011). From the regulation of peptidoglycan synthesis to bacterial growth and morphology. Nat. Rev. Microbiol. 10, 123-136. doi: 10.1038/nrmicro2677

Typas, A., Banzhaf, M. B., van den Berg van Saparoea B., Verheul, J., Biboy, J., Nichols, R. J., et al. (2010). Regulation of peptidoglycan synthesis by outermembrane proteins. Cell 143, 1097-1109. doi: 10.1016/j.cell.2010.11.038

Vollmer, W. (2012). Bacterial growth does require peptidoglycan hydrolases. Mol. Microbiol. 86, 1031-1035. doi: 10.1111/mmi.12059

Vollmer, W., Blanot, D., and de Pedro, M. A. (2008a). Peptidoglycan structure and architecture. FEMS Microbiol. Rev. 32, 149-167. doi: 10.1111/j.1574-6976.2007.00094.x

Vollmer, W., Joris, B., Charlier, P., and Foster, S. (2008b). Bacterial peptidoglycan (murein) hydrolases. FEMS Microbiol. Rev. 32, 259-286. doi: 10.1111/j.1574-6976.2007.00099.x

Zhao, H., Patel, V., Helmann, J. D., and Dorr, T. (2017). Don't let sleeping dogmas lie: new views of peptidoglycan synthesis and its regulation. Mol. Microbiol. 106, 847-860. doi: $10.1111 / \mathrm{mmi} .13853$

Conflict of Interest Statement: The authors declare that the research was conducted in the absence of any commercial or financial relationships that could be construed as a potential conflict of interest.

Copyright () 2019 Dörr, Moynihan and Mayer. This is an open-access article distributed under the terms of the Creative Commons Attribution License (CC BY). The use, distribution or reproduction in other forums is permitted, provided the original author(s) and the copyright owner(s) are credited and that the original publication in this journal is cited, in accordance with accepted academic practice. No use, distribution or reproduction is permitted which does not comply with these terms. 